\title{
Effects of Congruency on Bereitschaftspotential While Performing a Bimanual Motor Task
}

\author{
Meghan McGowan*, Camille Hémond-Hill*, Justine Nakazawa* \\ University of Victoria \\ meghanwmcgowan gmail.com
}

\begin{abstract}
The bereitschaftspotential (BP) - also known as the readiness potential - is a measure of brain activity that precedes voluntary movement by approximately one second in the supplementary motor area and the contralateral primary motor cortex. Motor task reaction time for bimanual task performance is affected by both the individual and the environment; however, it is unclear whether motor task reaction time (as measured via the BP) is significantly affected by congruency. A congruent motor task is an ipsilateral stimulus (e.g., a stimulus on the right is responded to by the right hand), and an incongruent task is a contralateral stimulus (e.g., a stimulus on the right is responded to by the left hand). Congruency is re-emerging as an important topic in motor learning as it may require different levels of cortical processing. The purpose of this study was to examine the effect of congruency on the BP. Participants were asked to complete the computer task, Keyboard Hero, where they pressed keys with both their left and right hands in response to discrete congruent and incongruent stimuli. A MUSE ${ }^{\mathrm{TM}}$ apparatus recorded brain activity $1000 \mathrm{~ms}$ prior to, and $1000 \mathrm{~ms}$ after each stimulus. Results from every participant for the incongruent and congruent trials were averaged and compared using a grand average waveform. Means of accuracy (how often participants pressed the key correctly) and BP for each condition were averaged and compared using a 95\% Confidence Interval (CI). Across congruent and incongruent conditions, a non-significant difference $(p>0.05)$ was found in $\mathrm{BP}(p>0.59)$, accuracy $(p>0.64)$, and $\mathrm{BP}$ within $-200 \mathrm{~ms}$ to $200 \mathrm{~ms}(p>0.31)$. BP and mean accuracy scores were not significantly different between congruent and incongruent conditions, which may be due to only minute differences in brain activity or due to the study's design. Further research should analyze individual variations of the present study, such as stimulus location, differences in the responding limb, correctness of responses, and the sensory modality being tested.

Keywords: Bereitschaftspotential; computer task; congruency; MUSE ${ }^{\mathrm{TM}}$; readiness potential
\end{abstract}

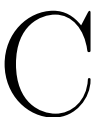
ongruency is re-emerging in the field of motor learning as a point of interest after an extended period without research. This is due to increased interest in the intricacies of training and learning for the most efficient use of instructional time. It is especially of interest in a competitive sport setting where the smallest difference in performance can lead to significant differences in results. Furthermore, studies of congruency are applicable in multiple settings: to those who wish to improve motor learning (e.g., coaches, physical educators), and to those who need to facilitate rehabilitation of movement (e.g., physiotherapists). If congruency indeed requires different levels of cortical processing that may affect accuracy and reaction time, then coaches and teachers should be notified to put more emphasis on practicing the skills that are more difficult for the brain to process. For example, a baseball that is thrown to a player's gloved side is more congruent than a throw to the player's non-gloved side. It would be useful for coaches to know

\footnotetext{
*We would like to thank Cameron Hassall for his irreplaceable continuous guidance, editing, and encouragement, Shikha Khurana for editing, Dr. Francisco Colino for his support and use of lab, and our fellow classmates for the provision of data.
} 
whether the congruent throw or the incongruent throw is more difficult to process cortically, and therefore which is more difficult to catch with the gloved hand. This would give coaches some insight as to which catching skills needs to be practiced more extensively; throws to the same side of the body or throws that require reaching across the body. Congruency studies could also be used in a clinical sense so that practitioners are better able to develop graded exercises for their patients. For example, a recovering stroke patient with hemiparesis may find it easier to complete congruent motor actions on their weakened side, so practitioners may decide to start strengthening exercises using solely congruent actions and then progress the activities to involve incongruent actions involving the opposite side of the body.

Motor task reaction time for bimanual task performance is typically affected by both the individual and the environment. It is unclear, however, whether or not congruency has an effect on optimal reaction time. A congruent task is one in which the location of the stimulus accords with the responding side of the body (i.e., the left side of the body responds to a stimulus on the left) (Serrien \& Spape, 1998). Although research shows that congruent conditions with a subject's hand can lead to improved reaction time, it remains uncertain whether incongruent conditions impair motor skills and increase reaction times. For example, if a stimulus appears on the left side of a display and the participant must respond with his or her right hand, then it is possible that the reaction time will increase, and thus more brain activity will be seen prior to executing the motor task. This uncertainty in the research prompted our study on whether congruency affects an individual's response time.

Prior to an individual's responding action, there are three processes that must occur: (1) stimulus identification; (2) response selection; and (3) response execution. Spatial attention plays a decisive role when responding to a stimulus - this phenomenon is termed the "Simon Effect" (O'Leary \& Barber, 1994). The Simon Effect hypothesizes that inhibition may play a role during the second stage, response selection, which causes a delay in an individual's response (O'Leary \& Barber, 1994). This effect may therefore suggest that reaction times are typically faster and frequently more accurate when a stimulus appears in the same relative location as the response location (O'Leary \& Barber, 1994).

Following the theory of the Simon Effect hypothesis, a study performed by Trapp, Lepsien, Sehm, Villringer and Ragert (2012) investigated the effects of reaction times when participants performed tasks with opposite hands. This task involved memorizing a sequence of button presses by the participants. The goal was to react as quickly as possible to a series of visual letters displayed on the screen. Trapp et al. (2012) hypothesized that the reaction times would increase when performing the task with opposite hands. For example, if participants started the experiment using their right hands and were then asked to use their left hands during the second trial, their reaction times would increase in the second trial. The results of the study demonstrated that when the subjects were forced to switch hands, ultimately creating incongruent spatial orientation in regard to one's hands, the reaction times for button presses were significantly slower. Also of importance, it was found that over the course of the study, subjects learned and adapted to the altered hand placement, thus leading to decreased reaction time for incongruent spatial orientation. This shows one factor that affects reaction time is the stimulus-response compatibility, the ability to learn through repeated trials (Fitts \& Seeger, 1953).

Another phenomenon, known as the "Stroop Effect," shows that when interference is present while performing a motor task, it is reflected in an individual's reaction time (O'Leary \& Barber, 1994). This effect is prevalent when individuals try to perform a task that is incongruent; for instance, naming the colour of ink of printed words while ignoring the words themselves (e.g., the word "red" written in blue ink instead of red ink). The results of such an incongruent task exhibit that individuals are slower in responding and are more error-prone in their responses than with 
congruent stimuli, such as saying the word "yellow" written in yellow ink (Braverman \& Meiran, 2010).

Similarly, in a study by Kato, Kizuka and Endo (2004), reaction times were observed for a congruent task and an incongruent task. Researchers used an electromyogram in order to analyze the results from both conditions. Electromyography measures the electrical activity of muscles at rest and during a muscle contraction prior to and upon presentation of a stimulus. Each participant was seated in front of a visual stimulus panel which consisted of five light-emitting diodes (LEDs). The panel contained a green LED fixation point that was located in the centre of the panel. There were two other green LED pre-cue lights located at a one-degree horizontal angle on both sides of the centre fixation point. The pre-cue lights would indicate whether the stimulus was going to be on the right or left side of the panel. Finally, there were two red LEDs located at a ten-degree horizontal angle from the centre. These red LEDs also indicated a "go" and "no-go" response - the "go" response was associated with a bright light and the "no-go" response was associated with a dim light. Participants were asked to depress two keys in order to maintain the fixation point. There was then either a left or a right stimulus light which was presented 0.5 to 1.5 seconds after the onset of a pre-cue light. The congruent task required participants to use a left response for a left stimulus and a right response for a right stimulus, whereas the incongruent task required the participants to use a left response for a right stimulus and vice versa. Finally, if a "go" stimulus light was presented the participants had to release the right-side key if the stimulus appeared on the right side. However, if a "no-go" stimulus was presented then subjects were told not to respond, and this meant that the participants needed to keep pressure on the keys. The results of the study showed that response preparation was a crucial factor for reaction time. Alongside these findings, it was also found that congruency was associated with faster reaction times. Kato et al. (2004) concluded that premotor time varied with the amount of response preparation and stimulus-response congruence.

Following the findings from these studies, it is apparent that there is some correlation with incongruent tasks and prolonged reaction times as well as increased brain activity prior to the participant's response. Thus, incongruent stimuli seem to have an effect on participants' preparation time. These discoveries were found using primarily EMG data collection (electromyography), which measures electrical responses of nerves in muscles, rather than electroencephalography, which measures brain wave activity.

In conjunction with the time allocated for response preparation during a motor task, the bereitschaftspotential (BP), also termed the "readiness potential," determines the amount of activity in the motor cortex as well as the supplementary motor area of the brain leading up to a voluntary muscle movement (Shibasaki \& Hallett, 2006). This cortical activity must gradually increase before the subject can perform the desired voluntary action. Thus, the BP is deduced to be an electrophysiological sign of motor planning, preparation, and initiation of the voluntary action (Shibasaki \& Hallett, 2006). These actions are present in any voluntary motor action, and include finger flexing, moving a limb, and even vocalizing a phenotype. Following the findings of the Kato et al. (2004) study, congruency should affect the amount of activity seen prior to the execution of a motor task. In our study, the BP will dictate whether or not there is greater or lesser amount of activity in the motor cortex and the supplementary motor area during a congruent or incongruent task.

Our study measured brainwave activity in order to determine BPs and investigated reaction times. Each participant had an electroencephalogram (EEG) MUSE ${ }^{\mathrm{TM}}$ device set up around their heads that followed their brainwave activity during both the congruent and the incongruent tasks. In order for the trial to be successful, participants must press the correct key on the keyboard with the correct hand at the correct time. Congruent data was defined as the responses from digits VI and $\mathrm{V}$ and incongruent data was defined as responses from digits II and III, both from the left and 
right hands. The digits IV and V were chosen as congruent for left and right because they were respectively the most left and most right parts of the body involved in this task. From EEG data, the BP was identified and analyzed. Data was analyzed in order to determine whether participants had larger BPs during the control or experimental trials. The current study provides a more in-depth look at aspects of congruency that have not yet been examined. This includes the isolation of spatial location to elicit a response, as opposed to past studies that require a separate decision to be made about hand position. The study was also smaller in scale; the visual fixation area is smaller, and different responses depend on the use of fingers as opposed to entire limbs. The results are therefore more applicable to fine motor skill tasks. Finally, the study used affordable EEG equipment that could potentially facilitate testing of large groups of participants in future studies of congruency. Testing larger groups may make subtle changes between conditions more clear and significant.

Ultimately, in accordance with both the Simon Effect and the Stroop Effect - as well as previous research including Trapp et al. (2012), and Kato et al. (2004) - we hypothesized that the data collected from the EEG monitors would demonstrate a larger BP during the incongruent trial than during the congruent trial. We also hypothesized that reaction time would be significantly longer in the incongruent task due to the relative additional processing time and would be demonstrated in greater amounts of "misses." The prediction put forward was that, since the response location did not correspond spatially with the subject's hand placement from initial learning trials, there would be more activity in the motor cortex and supplementary motor areas. Thus, there would be a greater readiness potential in the incongruent trials.

\section{Methods}

\section{Participants}

The participants $(N=30 ; 11$ male, 19 female; 4 left-handed, 26 right-handed; mean age $22.6 \pm 2.0$ years) of the experiment were undergraduate students at the University of Victoria (UVic) registered in the Exercise Science, Physical and Health (EPHE) program. The participants were healthy, active individuals with competent, unhindered brain function. This study was gender and sex inclusive. This was a novel task for the participants. Participation in this experiment was part of a university class requirement; however, participants gave consent in the beginning of the term, prior to data collection. The data collection room was dimly lit and a small amount of noise was present due to occasional conversations from other participants testing within the same room. Four participants partook in a pilot study, and the remaining 26 participants partook in testing. Data from both the pilot study and the testing protocol were used in the EEG analysis, but data from the pilot study were not included in the behavioural data analysis due to a change in the testing protocol. The new testing protocol included feedback concerning hits and misses to help guide future responses using a fixation cross that changed color depending on if the participant was accurate or not.

\section{Procedure and Apparatus}

\section{Apparatus}

The MUSE ${ }^{\mathrm{TM}}$ apparatus (InteraXon, Ontario, Canada) was originally designed to monitor the wearer's brain activity to give feedback on the meditation practice of the individual. This apparatus was reprogrammed using a unique computer program designed at UVic to show the desired brain activity for this experiment.

A single MUSE ${ }^{\text {TM }}$ device was turned on by pressing a start button. Using Bluetooth ${ }^{\circledR}$, the MUSE $^{\text {TM }}$ device was paired to a 2012 Apple iMac ${ }^{\circledR}$ (resolution: $1280 \times 800$, processor: $3.4 \mathrm{GHz}$ Intel 
Core i7) with an OS X El Capitan operating system. Horizontal and vertical screen lengths were $59.5 \mathrm{~cm}$ and $33.5 \mathrm{~cm}$ respectively. The pairing was complete when the corresponding MUSE ${ }^{\mathrm{TM}}$ identification (ID) number matched the MUSE ${ }^{\mathrm{TM}}$ number shown on the computer, and the Bluetooth icon on the computer was bolded. Multiple pairing attempts were necessary. Using the unique UVic experiment presented in MATLAB R2016b (MathWorks, Natick, MA), the correct coding and test subject's ID were entered. Once the program was ready to be run, the MUSE ${ }^{\mathrm{TM}}$ battery life was checked to ensure it could run for the entirety of the experiment. The program was started after the MUSE $^{\mathrm{TM}}$ was appropriately placed on the participant.

\section{Procedure}

Set-up. With the help of a research assistant, the participant was comfortably seated in a chair in an upright, relaxed position, 40-50 cm (approximately an arm's length) away from the computer, facing the computer screen with both hands resting on the keyboard. Both the keyboard and the computer were kept on the same desk (i.e., at the same height) directly in front of the participant so that the middle of the screen and keyboard were in line with the middle of the participant's body. The keyboard was placed in between the participant and the computer, 20-30 cm away from the base of the computer. The participant's knees, toes, and head were pointed forward, they sat with a neutral spine, and arms were flexed forward. The chair was adjusted so both of the participant's soles were fully in contact with the ground and resting comfortably. Ranges of participant and equipment positioning were given to accommodate for variations in participant dimensions and seating preference.

Long head hair on the test subject was tied back, and hair was tucked behind the participant's ears. The research assistant placed the MUSE ${ }^{\mathrm{TM}}$ apparatus on the participant's head with the inside of the convex part of the device in contact with the test subject's forehead, and the ear pieces behind the subject's ears. The MUSE ${ }^{\mathrm{TM}}$ was tightened, and water may have been added to ensure a solid connection on all four electrodes for accurate readings. Upon placing the MUSE ${ }^{\mathrm{TM}}$ on the participant's head, the program was started to check the quality of the MUSE ${ }^{\mathrm{TM}}$ connection to the participant. Initially an EEG appeared on the screen with four EEG waves corresponding to the four electrodes of the MUSE ${ }^{\mathrm{TM}}$ to the participant. At first, the four waves were red; however, the MUSE $^{\mathrm{TM}}$ was adjusted by the research assistant to ensure that all four waves were green before proceeding to data collection.

Once appropriate connection was established, the participant was directed to an instructional screen that explained the task, Keyboard Hero. The participant was instructed to press keys on the keyboard as a colored dot crossed the horizontal line. Participants were told not to hold the key for an extended period, but to only press the appropriate key one time as the dot crossed the horizontal line. Dots were small and circular $(1.3 \mathrm{~cm}$ diameter $)$, or large and oval shaped (length: 13 $\mathrm{cm}$, center width $1.3 \mathrm{~cm}$ ). In addition, the dots were blue or green, which corresponded to pressing the keys with their left hand or right hand respectively. Participants were instructed to place digits II-V of the left hand on keys R, E, W, Q respectively, and to place digits II-V of the right hand on keys U, I, O, P respectively. Participants were instructed to read the screen from left to right, meaning that a blue dot on the far left of the screen was to be pressed by digit $\mathrm{V}$ of the left hand (key Q), and that a green dot on the far left of the screen was to be pressed by digit II of the right hand (key U). In addition, participants were instructed to keep their eyes on a white cross in the center of the display at all times. The center of the cross was located $5.4 \mathrm{~cm}$ above the bottom horizontal line, and $29.45 \mathrm{~cm}$ from the left end of the screen. The cross turned cyan when a key was pressed correctly ("hit"), and red when a key was pressed incorrectly ("miss"). Prior to beginning the task, the participant was able to clarify the instructions with the research assistant. Once the 
participant understood the instructions, he or she was instructed to begin the task when ready.

Pilot study. Participants $(N=4)$ participated in a pilot study. The set-up procedure and data collection procedure were identical to the testing procedure except there was no fixation cross in the pilot study. Therefore, there was no hit or miss information provided to the participants.

Testing. The testing screen was black with four centered vertical white lines and one horizontal white line. The horizontal line was $8.5 \mathrm{~cm}$ above the bottom of the screen, and the vertical lines were $25.7 \mathrm{~cm}, 28.2 \mathrm{~cm}, 30.7 \mathrm{~cm}$, and $33.2 \mathrm{~cm}$, from the left edge of the screen, leaving a gap of $2.5 \mathrm{~cm}$ between each vertical line. Dots appeared centered on one of the four vertical lines, and dropped at a constant rate of $16 \mathrm{~ms}$ ( 0.2 degrees of visual angle for every screen refresh) towards the horizontal line. This rate allowed the participant a minimum of $1000 \mathrm{~ms}$ to make a response. Dots initially appeared $11.6 \mathrm{~cm}$ away from the horizontal line (Figure 8) or at the top of the screen $(25.2$ $\mathrm{cm}$ away from the horizontal line) (Figure 9). Dots would appear in a random sequence, either as a single dot (Figure 10) or multiple dots (Figure 11); each sequence was restricted to a single colour. Each dot was considered a trial, and 480 trials were completed in the task. When the participant believed that a dot was crossing the horizontal line, he or she pressed the corresponding key. If the dot was in fact on the horizontal line when the key was pressed, this was a "hit," and if the dot was not on the horizontal line when the key was pressed, or if the wrong key was pressed, this was a "miss." After each trial, the participant was given feedback which showed whether or not they were successful by demonstrating the cyan or red cross respectively. Participants were given two breaks for rest and also to check MUSETM connection quality. The two breaks were evenly dispersed one-third and two-thirds of the way through the experiment. Participants were told to take as long as they needed for rest; however, they were not permitted to remove the MUSE ${ }^{\mathrm{TM}}$ or stand up. Throughout the entirety of the experiment, the research assistant monitored the MUSE ${ }^{\mathrm{TM}}$ device to ensure the connection points were receiving data and the battery level was adequate. Upon completion of the task, the MATLAB R2016b (MathWorks, Natick, MA) with the Psychophysics Toolbox extension (Brainard, 1997) was exited and closed, and the device was removed from the participant. The MUSE ${ }^{\mathrm{TM}}$ device was unpaired from the computer and turned off.

\section{Data Collection}

Behavioural EEG data, as defined as congruent and incongruent data, were collected. For the purpose of this experiment, congruent data were defined as the responses from digits VI and V of the left and right hand, and incongruent data were defined as responses from digits II and III of the left and right hand. The digits IV and V were chosen as congruent for left and right because they were respectively the furthest left and furthest right parts of the body involved in this task. In addition, these digits responded to dots descending down the furthest left two vertical lines, or the furthest right vertical lines. Therefore, congruency was demonstrated as the stimulus on the left was responded to by the left side of the body and the stimulus on the right was responded to by the right side of the body. In comparison, the digits II and III of the left and right hands were chosen as incongruent responses as these digits responded to dots descending down the furthest right two vertical lines, or the two left vertical lines on the opposite side. Therefore, incongruency was demonstrated as the stimulus on the left was responded to by the right side of the body, and the stimulus on the right was responded to by the left side of the body.

Behavioural EEG data were recorded as hits and misses for a total of 480 trials. Hits were defined as pressing the correct key at the correct time. Correct keys were determined by the vertical line on which the stimulus descended and the colour of the dot; this determined which finger would be activated to press the key. The correct time was determined by the dot crossing the horizontal line; for it to be considered a "hit" the key must have only been pressed as the dot was crossing 
the horizontal line. The participant was alerted to the occurrence of a hit by the white fixation cross turning cyan. Misses were defined as (1) a key press that did not correspond to the correct colour on the screen; (2) a key press that did not correspond to the proper location of the vertical line on the screen (i.e., digit II of the right hand responding to a stimulus on the far right column during a congruent trial); and (3) a key press that was executed too early or too late according to the definition of a hit. The participant was alerted to the occurrence of a miss by the white fixation cross turning red.

The EEG data were collected and recorded via the MUSETM using a pre-established research sampling rate of $500 \mathrm{~Hz}$ (i.e., one sample was collected every two milliseconds, and no onboard data processing [InteraXon, Ontario, Canada]). The EEG data were collected through several electrodes on the MUSE ${ }^{\mathrm{TM}}$ that were located at points Fpz (reference electrode), AF7, AF8, TP9, and TP10. As EEG data were collected, they were directly transmitted via Bluetooth ${ }^{\circledR}$ into MATLAB R2016b (MathWorks, Natick, MA) with the Psychophysics Toolbox extension (Brainard, 1997). During the task, EEG data were recorded between $1000 \mathrm{~ms}$ prior to and $1000 \mathrm{~ms}$ after each response.

\section{Data Analysis}

The EEG data for each condition (congruent vs incongruent) were analyzed using a unique script coded in MATLAB R2016b (MathWorks, Natick, MA) and were filtered using a 0.1-15 Hz bandpass filter. Each trial was corrected in accordance to the baseline 1000-800 ms prior to each response; trials were excluded if they exceeded the change in voltage by $\pm 200 \mu \mathrm{V}$ relative to the baseline. For each participant, all data collection intervals for both conditions for each participant were averaged. Data collection intervals were plotted between $400 \mathrm{~ms}$ before and after the response. A grand average was then computed across all participants for each data collection interval for each condition. The grand averages for each condition were plotted against each other in a grand average waveform to determine the difference in the BP. Normally, the BP data in such studies are obtained from a central electrode located above the motor cortex; however, in this study, the MUSETM was placed on the scalp and took averages of electrodes from TP9 and TP10 regions. For the purpose of this experiment, the BP was defined as the mean voltage from $600 \mathrm{~ms}$ to $200 \mathrm{~ms}$ prior to each response at the pooled electrode TP9 and TP10. The mean BP for each condition was compared using a bar graph. A paired samples $t$-test was calculated for BP, comparing the congruent and the incongruent conditions to determine if there was an event related potential (ERP) difference.

Hit rates were averaged across all trials, where the program recorded a hit as positive one and a miss as negative one, demonstrating a hit ratio. For instance, an average of positive one implied that every trial was a hit and an average of negative one implied that every trial was a miss, whereas an average of zero was programmed when half the trials were hits and half were misses. Furthermore, if there was no button press, the score was not included in the results but was recorded as a zero in the program. The mean accuracy was calculated for every participant for both conditions. A grand average was then calculated to determine the accuracy for each condition. The means were then compared using a bar graph, and a paired samples $t$-test was conducted to determine if there was any significant variance between conditions.

A difference waveform comparing the voltage differences between the incongruent and congruent was calculated. Participant averages were then calculated for every data interval point for both conditions. Standard deviation and a $95 \%$ CI was then calculated from this average. A difference waveform with the $95 \%$ CI was then created.

Using the Grand Average Waveform, the time interval of the BP was determined. Using this smaller time interval, a second grand average waveform was calculated. The mean voltage for each condition was then compared using a bar graph. A paired samples $t$-test was then calculated for BP, 
comparing the congruent and the incongruent conditions to determine if there was an event related potential difference.

\section{Results}

Analyses focused on the participant's BP across the two different conditions: the congruent and incongruent spatial orientation tasks. There were three different categories of results which were deemed important: (1) EEG results identifying the mean BP across both conditions; (2) the behavioural results which established whether or not there were differences between the accuracy of button presses (hits vs misses) in both conditions; and (3) EEG results identifying the mean BP in the specific timeframe of $-200 \mathrm{~ms}$ to $200 \mathrm{~ms}$. The results examined all 480 trials of each participant in both the congruent and incongruent spatial orientations.

\section{EEG Results BP 1}

The first EEG results examined were taken in the time frame of $-800 \mathrm{~ms}$ to $700 \mathrm{~ms}$. When evaluating the mean $\mathrm{BP}$ across the two conditions, congruent and incongruent spatial orientations, results yielded $-1.37 \mu \mathrm{V}, 95 \% \mathrm{CI}[-2.13,-0.60]$ for congruent and $-1.19 \mu \mathrm{V}, 95 \% \mathrm{CI}[-1.98,-0.40]$ for incongruent, as displayed in Figure 2 and Figure 3. A $t$-test revealed that the difference in means yielded an insignificant result of $t(29)=0.54, p=0.59$.

\section{Behavoural Results}

The precision of button presses by participants was calculated by identifying the mean accuracy across the two conditions, congruent and incongruent, as seen in Figure 4. Results yielded 0.53, $95 \%$ CI $[0.43,0.61]$ and $0.54,95 \%$ CI $[0.44,0.64]$ respectively. For these conditions, the use of a $t$-test revealed an insignificant result of $t(25)=0.47, p=0.64$.

\section{EEG Results BP 2}

The mean of the BP in the particular timeframe of $-200 \mathrm{~ms}$ to $200 \mathrm{~ms}$, across the two conditions was calculated to investigate whether or not there was a significant difference between the two conditions in a more specific time frame. As illustrated in Figure 6 and Figure 7, a result of -2.08 $\mu \mathrm{V}, 95 \% \mathrm{CI}[-3.02,-1.15]$ for congruent and $-1.77 \mu \mathrm{V}, 95 \% \mathrm{CI}[-2.70,-0.84]$ for incongruent was found. Again, to determine whether these differences were significant a $t$-test was conducted. The $t$-test revealed an insignificant result of $t(29)=1.04, p=0.31$.

\section{Discussion}

The grand average waveform of mean voltages revealed that participant brain activity in congruent and incongruent conditions followed a very similar pattern as seen in Figure 2. The average BP across the entire duration of one trial was slightly larger in the congruent condition compared to the incongruent condition as can be seen in Figure 3; however, this difference was not statistically significant. Similarly, congruent condition voltages were larger negatively than incongruent condition voltages in the isolated time-frame of $-200 \mathrm{~ms}$ to $200 \mathrm{~ms}$ as seen in Figure 7; however, these results were also not statistically significant. A difference waveform as seen in Figure 5 revealed that there was a significant difference in BPs earlier than anticipated, between $-700 \mathrm{~ms}$ and $-400 \mathrm{~ms}$. The behavioural data across both conditions was almost the same as seen in Figure 4, and there was no significant difference found in the mean accuracy between conditions. 
The researchers had hypothesized that BPs would be larger negatively for the incongruent condition compared to the congruent condition due to the Simon Effect described by O'Leary and Barber (1994); however, the findings of the current study do not support the hypothesis considering the lack of significant results. Contrary to the hypothesis, BPs for the congruent condition tended to be larger negatively than BPs for the incongruent condition. Additionally, electrical brain activity followed a very similar pattern between conditions (as seen in Figure 2), which indicates that participants were putting in effort to complete the task accurately, but also demonstrates a lack of difference in responses. The second hypothesis stated that mean accuracy of response time would be greater for the congruent condition compared to the incongruent condition due to the increased reaction time necessary for incongruent responses (Kato et al., 2004); however, the findings of the current study do not support the hypothesis considering the lack of significant results. This could be due to a genuine lack of an effect or due to the study's design. Previous studies consistently found that incongruent conditions hindered performance and caused a larger effect than congruent conditions (Forster \& Pavone, 2008; Kato et al., 2004; Wang, Du, He, \& Zhang, 2014). Considering the wealth of studies finding a significant difference between congruent and incongruent conditions, it is likely that the current study had issues relating to its design that complicated finding a significant result. It is possible that this study found a moderating factor that alters the common tendencies of the Simon Effect (e.g., the Keyboard Hero task), but new studies would need to be performed to isolate these variables.

The protocols of the present study involved only subtle and simple differences in response stimuli and in response actions. The difference between congruent and incongruent stimuli were separated by only a few centimeters, and the differences in responses was between fingers on the same hand. Past studies of congruency tend to use much larger distances between stimuli and separate responses between entire limbs (Wang et al., 2014). Because of this, it may be that the differences recorded were too small to be significant with the sample size used. Increasing the sample size could help highlight these subtle differences; however, in a practical sense, it may be more beneficial to change the design of the study to see larger differences in responses and be more applicable to other settings. In an EEG study analyzing BP, Forster and Pavone (2008) found that correctly executed responses scored higher negatively in incongruent trials compared to congruent trials in the $-200 \mathrm{~ms}$ to 0 ms range, but that incorrectly executed responses scored lower negatively in incongruent trials compared to congruent trials in the $0 \mathrm{~ms}$ to $200 \mathrm{~ms}$ range. Considering that the relative magnitudes of the BPs for congruent and incongruent trials depended on if the trial was a correct or incorrect response, the results of the current study may have been "washed out" since correct and incorrect responses were analyzed together.

The location of the electrodes may also pose a threat to validity as it was found that the largest ERP waveforms were recorded over frontocentral electrode sites (Forster \& Pavone, 2008). These sites would relay feedback from the supplementary motor area (SMA) and the primary motor cortex (M1), both of which are the cortical sites responsible for the early and late components of the BP respectfully (Smith \& Staines, 2012). The MUSE ${ }^{\mathrm{TM}}$ device does not have receptor sites that cover these areas of the scalp, meaning critical differences in activity may have been lost relating to the difference in BPs if the primary site of the decision-making process was not being monitored. Improved analysis of these brain regions would also be beneficial to study early BPs, considering that differences in voltages between $-2 \mathrm{~ms}$ and $-500 \mathrm{~ms}$ are implicated with preparatory motor planning in the SMA. Additionally, there were significant differences in early BPs of the current study as seen in the difference waveform (Figure 5). The task may demonstrate more neurological differences in the early BP, which indicates that the focus of the analyses should be shifted to an earlier time period in the EEG analysis. This could imply that this specific task requires more changes in preparatory motor planning from the SMA as opposed to general movement planning in 
the M1 (Smith \& Staines, 2012).

The findings of the current study seem to contradict past work that tests the Simon Effect; however, differences in distances between the responding limb and the stimulus screen have been shown to vary the magnitude of the Simon Effect (Wang et al., 2014). Therefore, the current study may not contradict Simon Effect study findings; it could be that the distance between the responding hand and the stimulus was not optimal and therefore did not yield a significant result. Larger distances between response sites have shown more significant results (Wang et al., 2014), indicating that it may have been beneficial to increase the distance between stimuli and responding limbs in a future variation of the current study. The previously mentioned study by Kato et al. (2004) found that incongruent response times were longer using EMG technology to record reaction time of a limb. EMG may be a better indicator of delayed response time compared to EEG, or the delay in response time could only be significant when it is an entire limb that is incongruent to the stimulus as opposed to digits on a single limb. A study measuring response times using digits II and III of each hand on a keyboard found that response times were improved when there was a simple transformational rule applied to relate the stimulus location to the button to be pressed (Read \& Proctor, 2004). This finding is consistent with the current study as the four keys to be pressed aligned logically with the stimuli on the screen, so there was no need for extra processing time considering the easily learned pattern. With regard to these past findings, a test of congruency relating to BPs and accuracy may have been better observed if the stimuli directed at each limb (i.e., the left and the right hands) had been switched, or if the organization of stimuli had been more random for each finger across trials. The three variations mentioned above, namely (1) increasing the distance between visual stimuli; (2) changing the data recording to EMG; and (3) randomizing the distribution of the response sites may all be useful in enhancing the Simon Effect to achieve a significant result.

There is also a series of similar research studies on the Crossmodal Congruency Effect (CCE), which is defined as the difference in reaction time when stimuli across different sensory modalities, such as touch and visuals, are congruent or incongruent (Forster \& Pavone, 2008; Sengül et al., 2012). They found that reaction times tend to be slower when sensory modality stimuli are incongruent or opposite to the responding side. The incongruent sensory stimulus is considered a distractor to proper responses (Forster \& Pavone, 2008; Sengül et al., 2012). This could imply that congruency may only have a significant effect when multiple sensory modalities are involved that may distract from responding, or that the distraction of experiencing an additional sensory stimulus that encourages an incorrect response (incongruent trial) produces a larger and more discernible effect. The visible effect may be due to the increased level of complexity that multiple modalities add to the task. Considering that the current study was using solely visual stimuli, it may be that there is minimal to no significant effect of congruency on response time when only one sensory modality is involved, and that the effects of the current study may be similar to CCE studies, but are more difficult to measure.

The current study contributes further insight into the differences in cortical planning and accuracy in response time of congruent and incongruent stimuli. Specifically, the study highlights the importance of (1) isolating certain variables including correct and incorrect responses; (2) further analysis of early BPs; and (3) modifying the design to reflect larger and more applicable differences in responses relating to congruency. The results of the current study were insignificant and were therefore not consistent with the findings of previous literature (Forster \& Pavone, 2008; Kato et al., 2004; Wang et al., 2014); however, these inconsistencies may have been due to differences in design. Future studies could isolate congruency tests that target different sensory modalities such as touch and sound to determine whether congruency has a larger effect on some senses compared to others according to the CCE effect. Measurement difficulty may also be due to the MUSE ${ }^{\mathrm{TM}}$ apparatus. 
The MUSE ${ }^{\mathrm{TM}}$ headset has four sensors, none of which cover the dorsal scalp. Considering that movement planning was the main focus of the study, and that such cortical processing tends to occur beneath more dorsal areas of the skull, some critical brain activity may have been missed by the MUSE ${ }^{\mathrm{TM}}$. Future studies may benefit from using a full EEG cap with electrodes that cover the SMA and the M1, and may be better at detecting subtle changes in brain activity in the related areas. Another method that may be used to strengthen the findings would be to differentially compare congruency and incongruency of entire limbs as opposed to different fingers on a hand. Past congruency studies that investigated the difference in hand responses and foot responses tended to yield more significant results. Results may also be more significant if correct and incorrect responses are analyzed separately; this minimizes the chances of "washing out" effects that may be opposite to each other depending on the feedback of the trial. Overall, these changes to design may yield more significant results with bigger differences between congruent and incongruent trials that are more useful and applicable to teaching and clinical settings.

\section{Conclusion}

Previous studies looked at the impact of congruency of stimuli and responses in gross motor actions and across multiple modalities on the stimulus identification, response selection and response execution cycle of the Simon Effect. The current study aimed to find a difference in the level of cortical processing and the accuracy in response time relating to congruent and incongruent actions in a novel computer game task. The task used fine motor skills and isolated vision as a single modality. It was found that there was no significant difference in EEG activity or response accuracy between conditions. This challenges previous findings about the level at which congruency has an effect of cortical processing and performance, and the specificity at which findings are significant in terms of stimulus location, differences in the responding limb, correctness of responses, and the sensory modality being tested. 


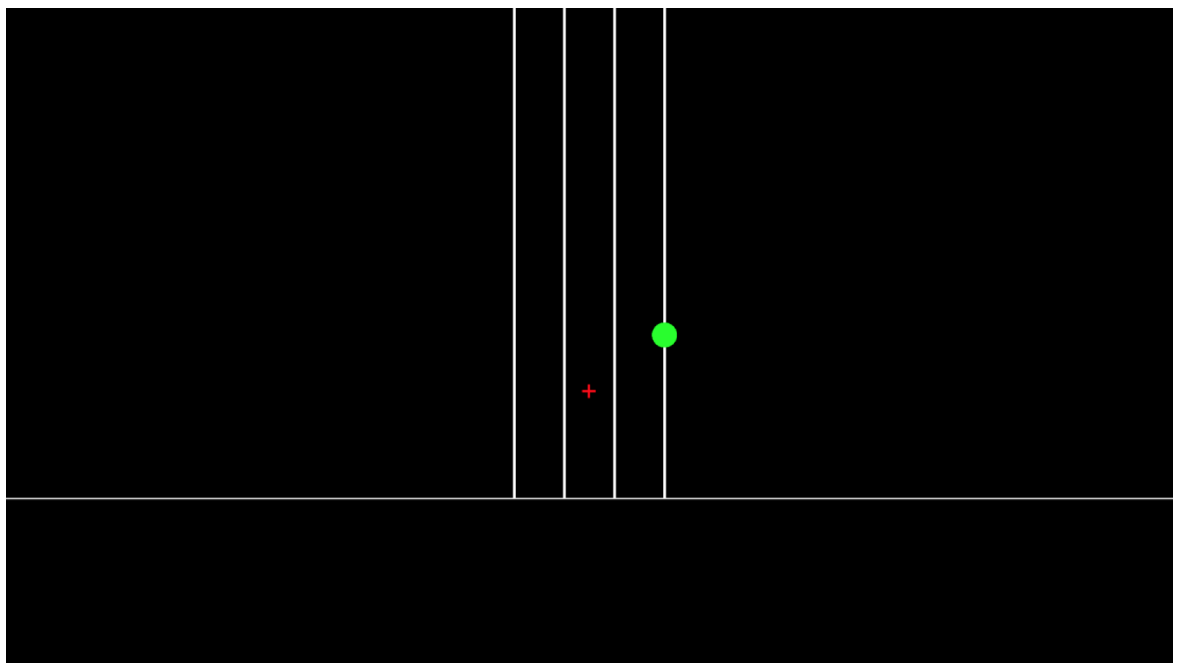

Figure 1: Screenshot of testing screen with a single trial. The fixation cross is red indicating that the previous trial was a miss.

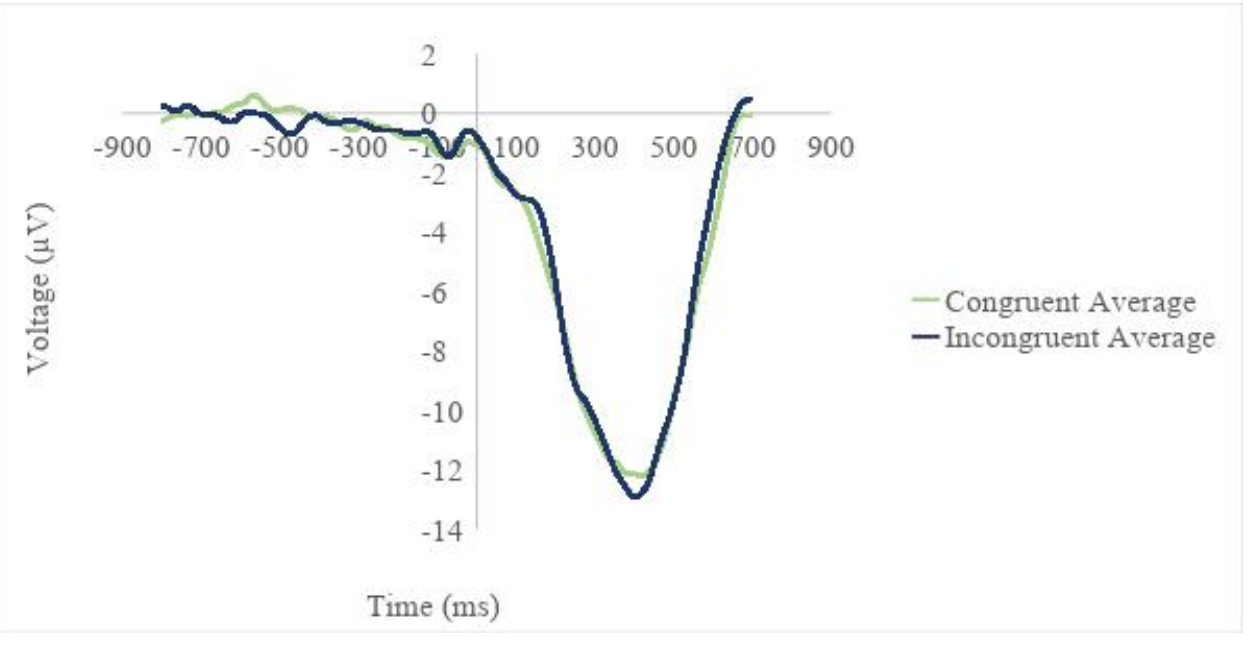

Figure 2: Grand average waveform comparing mean voltages of electroencephalogram recorded brain activity across participants at each time point for both the congruent and incongruent spatial orientation conditions. The waveform displays results from a timeframe of $-800 \mathrm{~ms}$ to $700 \mathrm{~ms}$ relative to initiation of the keypress by the participant. 


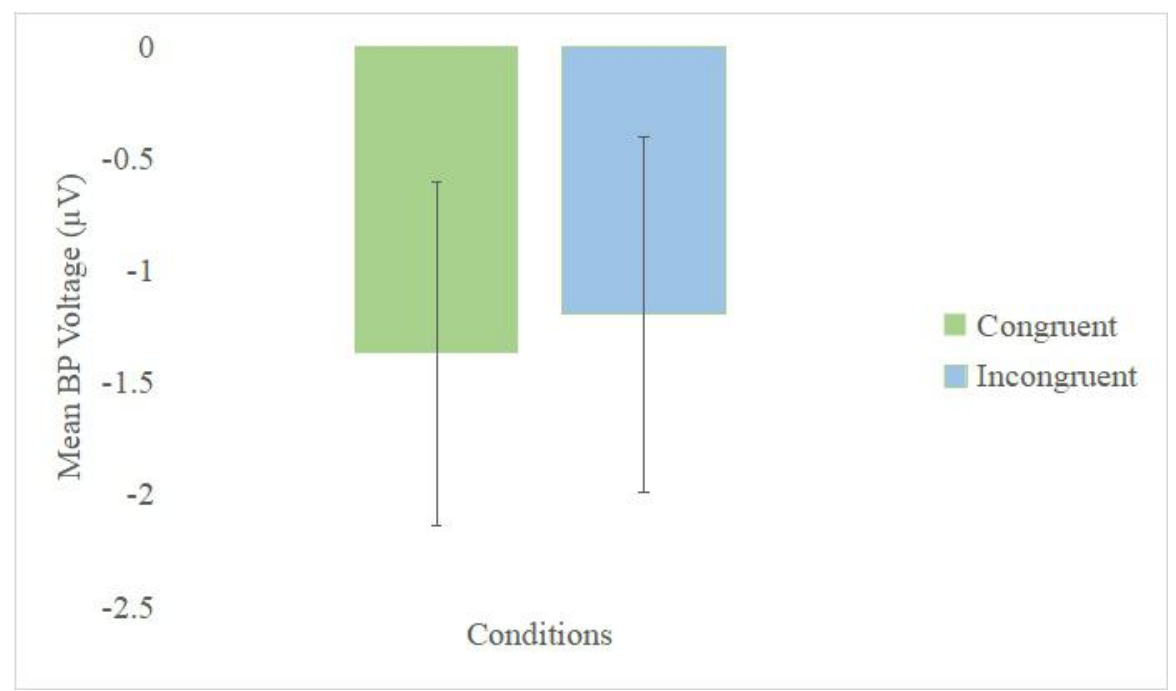

Figure 3: Average bereitschaftspotential voltage of electroencephalogram recorded brain activity of the congruent and incongruent spatial orientation conditions in the time frame of $-800 \mathrm{~ms}$ and 700 ms with 95\% confidence interval error bars.

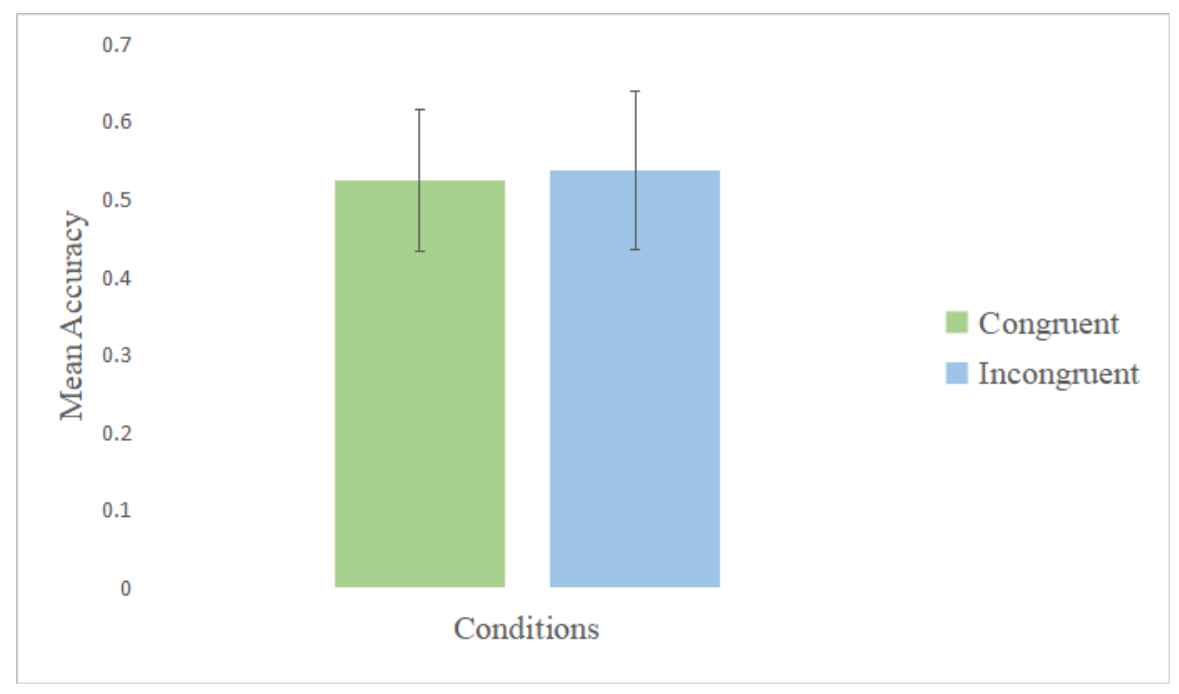

Figure 4: Average response accuracy of keypress by participants for the incongruent and congruent spatial orientation (i.e., the precision of button presses - the correct key on the keyboard with the correct hand at the correct time with $95 \%$ confidence interval error bars). 


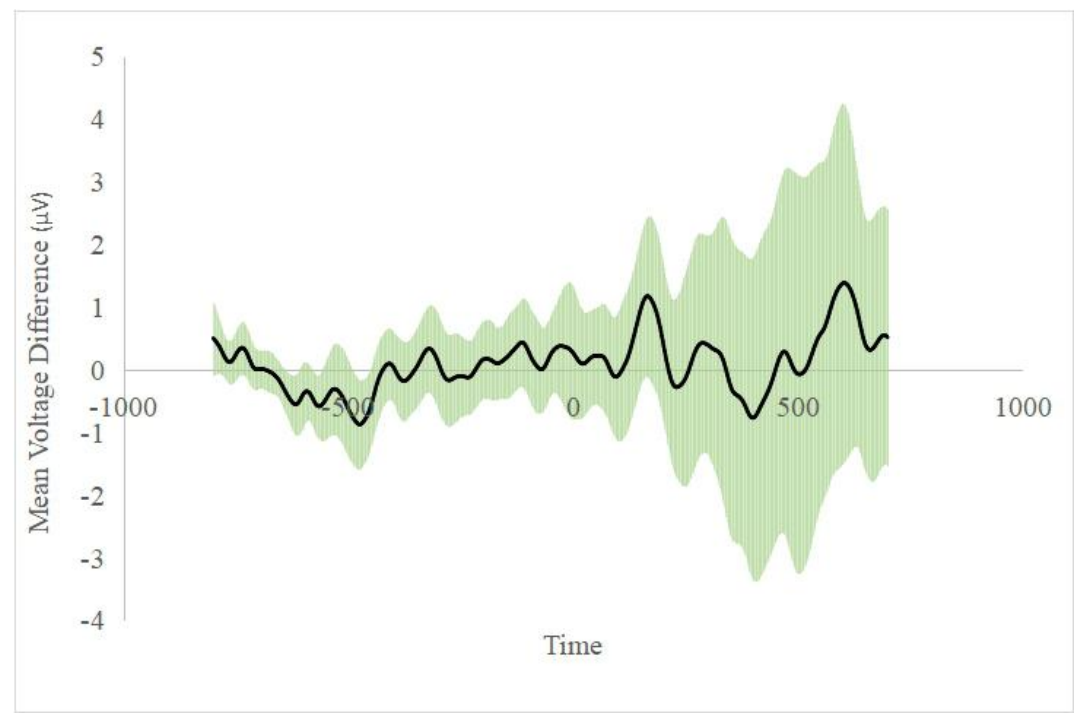

Figure 5: A difference waveform comparing the mean voltage of electroencephalogram recorded brain activity across time. The waveform demonstrates results of both the incongruent spatial orientation condition to the mean voltage of the congruent spatial orientation condition using error bars with a $95 \%$ confidence interval.

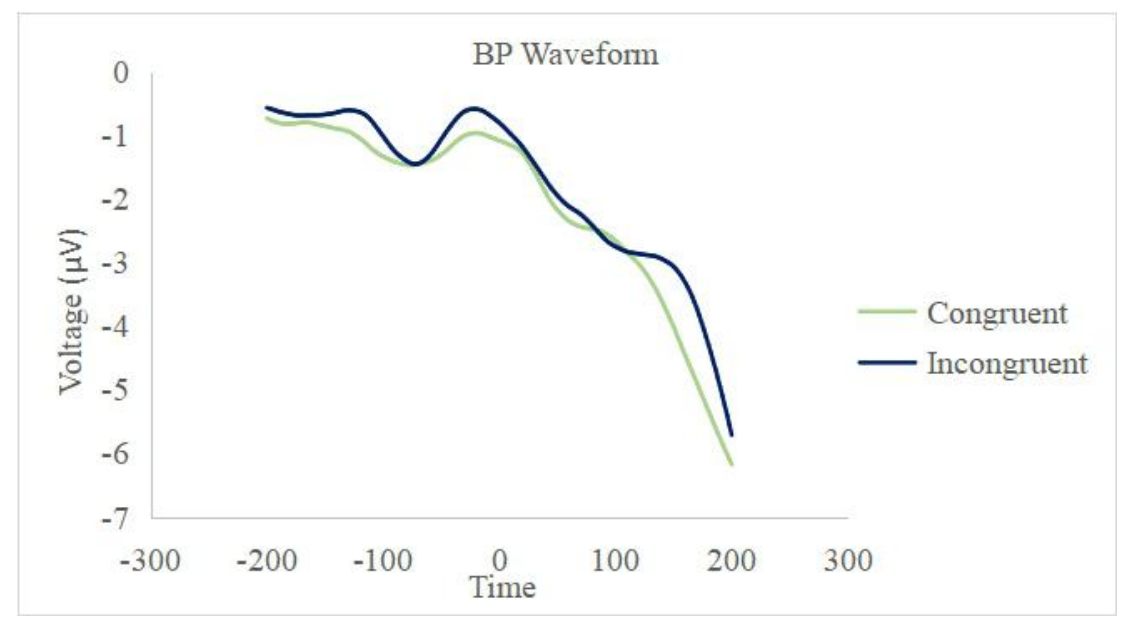

Figure 6: Grand average waveform comparing mean voltages of electroencephalogram recorded brain activity across participants at each time point for both the congruent spatial orientation and incongruent spatial orientation conditions. This waveform displays results from a timeframe of $-200 \mathrm{~ms}$ to 200 ms relative to initiation of the keypress by the participant. 


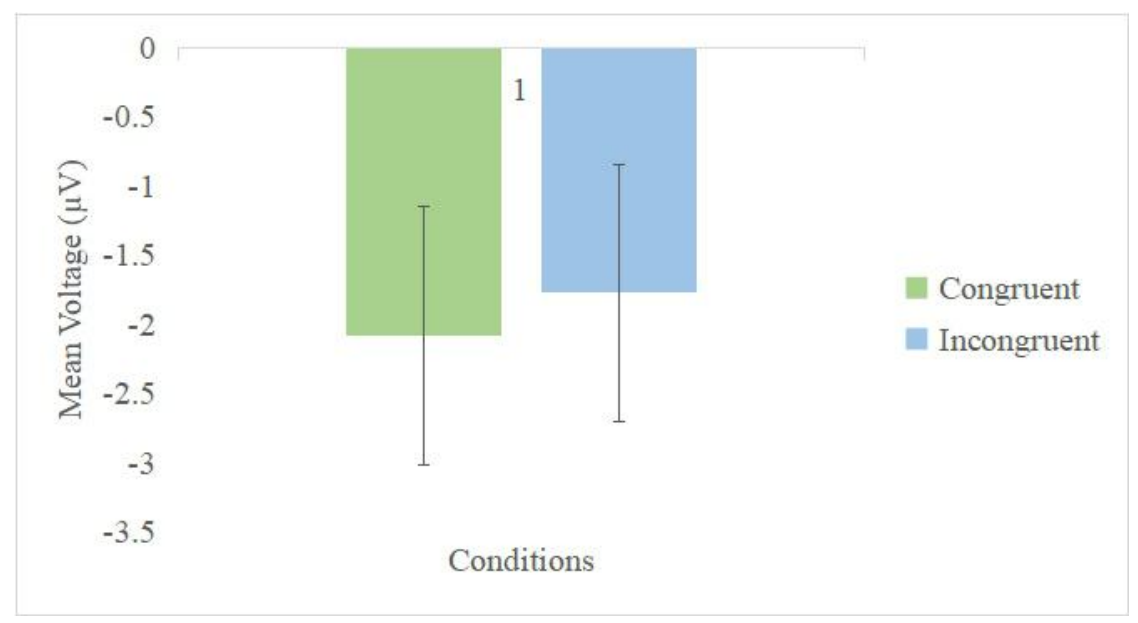

Figure 7: Average bereitschaftspotential voltage of electroencephalogram recorded brain activity for both the congruent spatial orientation and incongruent spatial orientation conditions with $95 \%$ confidence interval error bars. The figure portrays results from a timeframe of $-200 \mathrm{~ms}$ to $200 \mathrm{~ms}$ relative to initiation of the keypress by the participant.

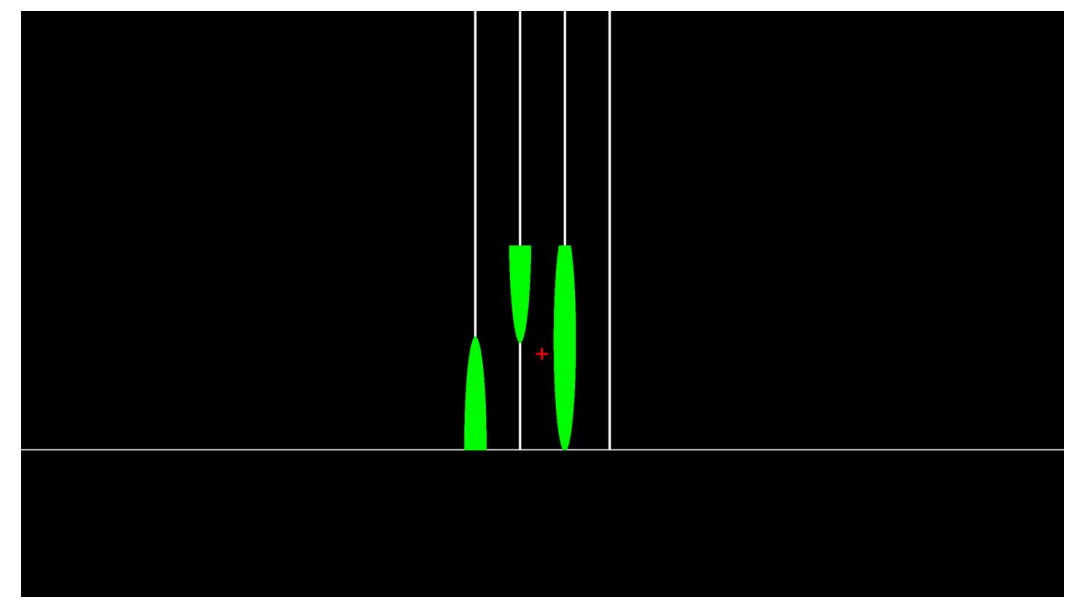

Figure 8: Sequence of large green dots appearing $11.6 \mathrm{~cm}$ above the horizontal line. 


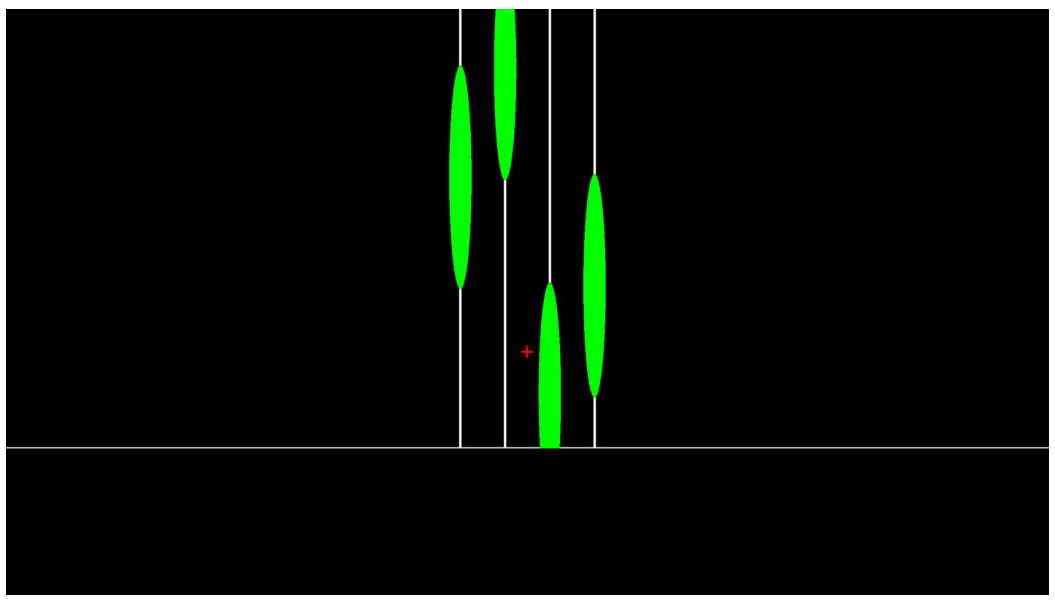

Figure 9: Sequence of large green dots appearing at the top of the screen.

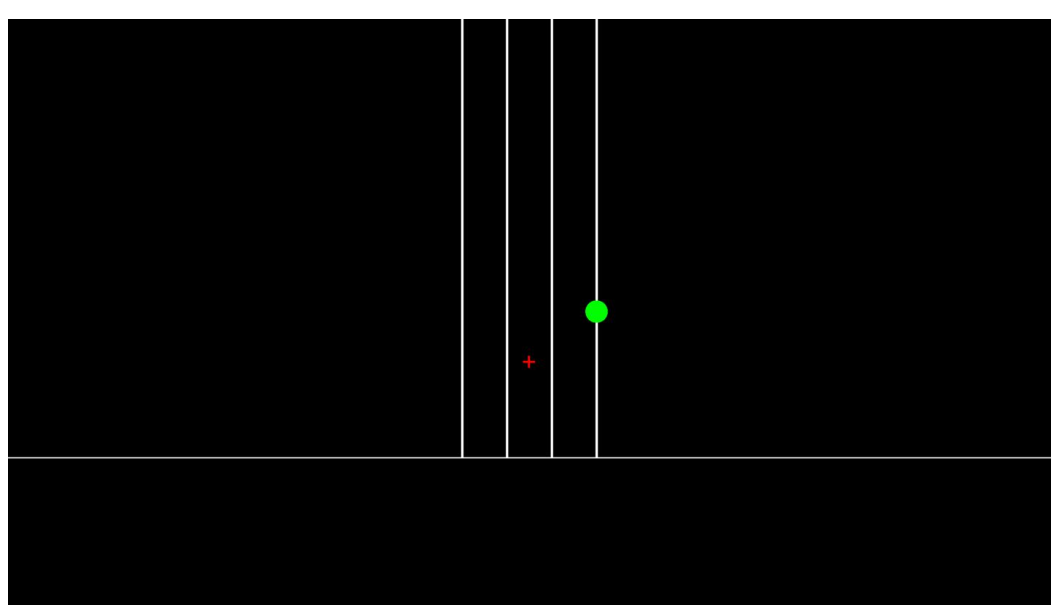

Figure 10: Sequence of single small green dot.

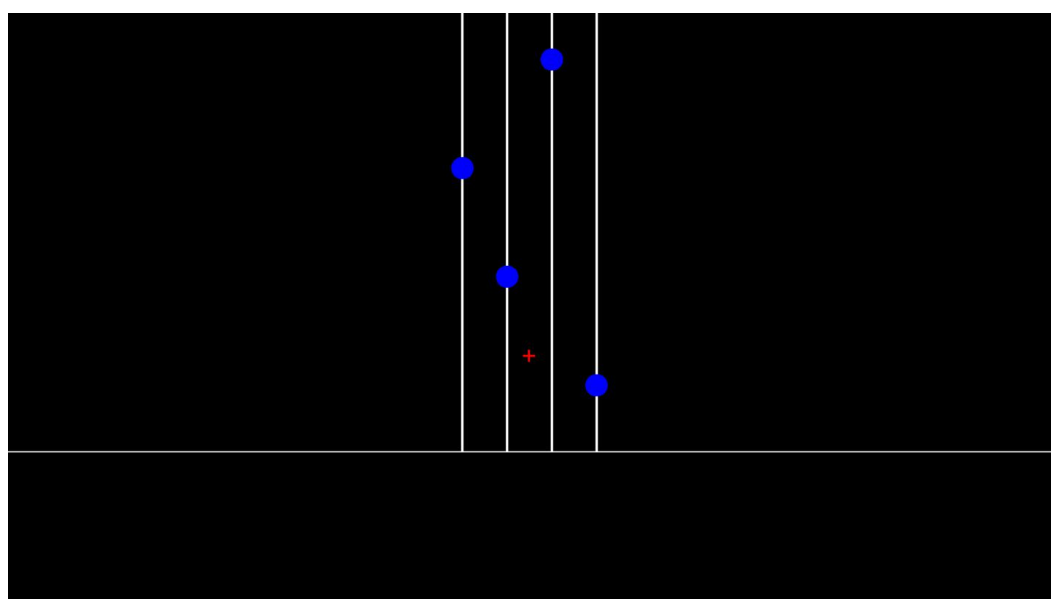

Figure 11: Sequence of multiple small blue dots. 


\section{References}

Braverman, A., \& Meiran, N. (2010). Task conflict effect in task switching. Psychological Research, $74,568-578$. doi: 10.1007/s00426-010-0279-2

Fitts, M., \& Seeger, C. M. (1953). S-R compatibility: spatial characteristics of stimulus and response code. Journal of Experimental Psychology, 46, 193-210.

Forster, B., \& Pavone, E. F. (2008). Electrophysiological correlates of crossmodal visual distractor congruency effects: Evidence for response conflict. Cognitive, Affective, \& Behavioural Neuroscience, 8(1), 65âĂŞ73. https://doi.org/10.3758/CABN.8.1.65

Kato, Y., Kizuka, T., \& Endo, H. (2004). Response preparation and stimulus-response congruence to fractionating reaction time of upper and lower limbs. Perceptual and Motor Skills, 99(1), 1926. http://journals.sagepub.com/doi/pdf/10.2466/pms.99.1.19-26 O'Leary, M., \& P. Barber. (1994). Stimulus congruence and the Simon effect. Psychological Research 56 (3), 196-202.

Read, L. E., \& Proctor, R. W. (2004). Spatial stimulus-response compatibility and negative priming. Psychonomic Bulletin \& Review, 11(1), 41-48. Retrieved from https://link-springercom.ezproxy.library.uvic.ca/content/pdf/10.3758\%2FBF03206458.pdf

Sengül, A., Van Elk, M., Rognini, G., Aspell, J. E., Bleuler, H., \& Blanke, O. (2012). Extending the body to virtual tools using a robotic surgical interface: Evidence from the crossmodal congruency task. PLoS ONE, 7(12), 1-9. https://doi.org/10.1371/journal.pone.0049473

Serrien, D., \& Spape, M. (1998). The role of hand dominance and sensorimotor congruence in voluntary movement. Experimental Brain Research 199(2), 195-200. Doi: 10.1007/s00221000-1998-8

Shibasaki, H., \& Hallett, M. (2006). What is the bereitschaftspotential?. Clinical Neurophysiology 117, 2341-2356. doi: 10.1016/j.clinph.2006.04.025

Smith, A. L., \& Staines, W. R. (2012). Externally cued inphase bimanual training enhances preparatory premotor activity. Clinical Neurophysiology, 123(9), 1846-1857. https://doi.org/10.1016/j.clinph.2012.02.060

Trapp, S., Lepsien, J., Sehm, B., Arno, V., \& Ragert, P. (2012). Changes of hand switching costs during bimanual sequential learning. Plos one $7(9)$.

Wang, X., Du, F., He, X., \& Zhang, K. (2014). Enhanced spatial stimulus-response mapping near the hands: The Simon Effect is modulated by hand-stimulus proximity. Journal of Experimental Psychology: Human Perception and Performance, 40(6), 2252-2265. https://doi.org/10.1037/a0038140 